\title{
Block Dissection of Inguinal Lymph Nodes
}

National Cancer Institute

\section{Source}

National Cancer Institute. Block Dissection of Inguinal Lymph Nodes. NCI Thesaurus.

Code C51620.

Surgical removal of multiple adjacent, frequently adherent, inguinal lymph nodes. 